(2) Open Access Full Text Article

\title{
Synergistic antitumoral efficacy of a novel replicative adenovirus SG6I I-PDCD5 and daunorubicin in human leukemic cells
}

This article was published in the following Dove Press journal:

OncoTargets and Therapy

\author{
Ya-Lan Zhou' \\ Qiu-Mei Yao' \\ Jiao Zhou' \\ Yan Chang' \\ Jin-Lan $\mathrm{Li}^{\mathrm{I}}$ \\ Ya-Zhe Wang' \\ Hong-Ping $\mathrm{Wu}^{2}$ \\ Yu-Hong Chen' \\ Yan-Rong Liu' \\ Xiao-Jun Huang' \\ Guo-Rui Ruan' \\ 'Beijing Key Laboratory of \\ Hematopoietic Stem Cell \\ Transplantation, Peking University \\ People's Hospital and Institute \\ of Hematology, Beijing, China; \\ ${ }^{2}$ Laboratory of Viral and Gene \\ Therapy, Eastern Hepatobiliary \\ Surgical Hospital, Second Military \\ Medical University, Shanghai, China
}

Correspondence: Guo-Rui Ruan Beijing Key Laboratory of Hematopoietic Stem Cell Transplantation, Peking University People's Hospital and Institute of Hematology, II Xi-Zhi-Men South Street, Beijing 100044, China

Tel +861088324672

Fax +861088324672

Email ruanguorui@pkuph.edu.cn
Background: Daunorubicin is a traditional chemotherapeutic agent that plays a pivotal role in leukemia therapy. However, the dose-related toxicity remains a considerable challenge. The apoptosis-regulating gene, $P D C D 5$, is downregulated in various tumors, including leukemias, and may provide a potential target for the diagnosis and treatment of leukemia. The purpose of this study was to construct a triple-regulated oncolytic adenovirus carrying a PDCD5 gene expression cassette (SG611-PDCD5) and explore the combined antitumor efficacy of SG611PDCD5 in combination with low dose daunorubicin on leukemic cells.

Materials and methods: A variety of leukemic cell lines, including K562, MEG-01, KG-1a, HL-60, SUP-B15, and BV-173, were cultured according to the providers' instructions. The insertion and orientation of all recombined plasmids were confirmed by restriction enzyme digestion and PCR. The tumor-selective replication of the constructed conditionally replicating SG611-PDCD5 and its antitumor efficacy in combination with daunorubicin were characterized in leukemic cell lines in vitro and in a nude mouse xenograft model. Cell viability was detected using cell-counting kit-8. Apoptosis was detected in whole living cells using flow cytometry and in paraffin-embedded tumor tissues using a terminal deoxynucleotidyl transferase dUTP nick end labeling (TUNEL) assay.

Results: The triple-regulated CRAd carrying SG611-PDCD5 and nude mouse xenograft models of K562 cells were successfully constructed. In vitro treatment with SG611-PDCD5 in combination with low-dose daunorubicin elicited more potent anti-proliferative and proapoptotic effects in leukemic cells in a dose-dependent manner. The Chou-Talalay analysis revealed synergistic anti-proliferative effects in all of the above cell lines. In the nude mice xenograft model, the tumor size in the control, daunorubicin, SG611-PDCD5, and combined treatment groups on day 10 were $170.1 \pm 47.8,111.9 \pm 81.1,60.7 \pm 12.3$, and $33.2 \pm 17.5 \mathrm{~mm}^{3}$, respectively (all $P<0.05$ ). The results of the TUNEL assay showed significantly more apoptotic cells in the SG611-PDCD5 plus daunorubicin group than in the SG611-PDCD5 or daunorubicin groups alone $(25 \pm 0.82$, 12.5 \pm 2.27 , and $7.8 \pm 2.67$ apoptotic cells/field, respectively) $(P<0.05)$.

Conclusion: The findings suggest that combined treatment with SG611-PDCD5 and daunorubicin may be a promising strategy for enhancing chemosensitivity and thus lowering the dose-related toxicity of daunorubicin in leukemia therapy.

Keywords: $P D C D 5$ gene, oncolytic adenovirus, leukemia, gene therapy

\section{Introduction}

Daunorubicin, a traditional chemotherapeutic agent, inhibits cancer cells by inhibiting their proliferation and/or inducing apoptosis, and it plays a pivotal role in leukemia therapy. Unfortunately, the dose-related toxicity, especially cardiotoxicity, caused by daunorubicin limits its clinical use and remains a considerable challenge for clinicians. ${ }^{1-3}$ 
Apoptosis-related genes such as $P 53,{ }^{4} P 73,{ }^{5}$ and $B A X^{6}$ have been used in cancer treatment, and studies have reported that the proteins encoded by these genes can induce apoptosis alone or synergistically, thereby enhancing the effects of traditional chemotherapeutics. ${ }^{7}$ The apoptosis-regulating gene PDCD5, also designated TF-1 cell apoptosis-related gene-19, has been identified in TF-1 cells by the Laboratory of Medical Immunology of Beijing Medical University, and it is downregulated in various tumors, including acute (AML) and chronic myeloid leukemia (CML). Moreover, a negative correlation has been observed between the relative level of PDCD5 and BCR/ABL expression in all CML patients and those in the advanced phase, suggesting that reduced $P D C D 5$ expression may play an important role in the mechanism of cancer pathogenesis and progression. ${ }^{8}$

Advances in genetic modification and the use of oncolytic viruses have formed the basis of numerous clinical trials on cancer therapies, some of which have shown promise. Su et al ${ }^{9}$ previously constructed a mono-regulated conditionally replicative adenovirus (CRAd) (CNHK300), a dual-regulated CRAd (CNHK500), ${ }^{10}$ and a triple-regulated CRAd (SG600) ${ }^{7}$ on the basis of the two common characteristics of most cancers, namely, the relatively high telomerase activation and hypoxic environment. ${ }^{11,12}$ Since then, rapid developments have been achieved in the field of CRAds. Given these advances and the hypothesis that the PDCD5 gene plays an important biological role in leukemogenesis, we constructed a triple-regulated CRAd carrying the PDCD5 gene expression cassette, SG611-PDCD5. In this cassette, the E1a gene with 24 nucleotides deleted from the CR2 region is controlled by the human telomerase reverse transcriptase (hTERT) promoter, $E 1 b$ gene expression is directed by the hypoxia response element (HRE), and the PDCD5 gene is controlled by the cytomegalovirus promoter. This innovative design makes this CRAd relatively selective with potent antitumor efficacy, as demonstrated with several leukemic cell lines (eg, K562, MEG-01, and SUP-B15), and the antitumor efficacy has been proved to be more potent than that of the PDCD5 protein or the replication-defective oncolytic adenovirus Ad-PDCD5. ${ }^{13}$

In the present study, we investigated the combined antitumoral effect of SG611-PDCD5 and daunorubicin in vitro and in vivo experiments. We found that the efficacy of SG611-PDCD5 and low-dose daunorubicin was significantly more potent at inhibiting the proliferation of and inducing apoptosis in human leukemic cell lines in vitro as well as in human leukemic cells xenografted in nude mice in a dosedependent manner. Our findings may lead to the development of new strategies for effective leukemia treatment and may reduce systemic toxicity.

\section{Materials and methods \\ Cell lines and culture conditions}

The human chronic myelogenous leukemic cell line K562 and human megakaryoblastic leukemic cell line MEG-01 were kindly provided by Cell Bank Shanghai Institute of Cell Biology (Shanghai, China). The human acute myeloid leukemic cell line KG-1a, human acute promyelocytic leukemic cell line HL-60, human acute lymphoblastic leukemia cell line BV-173, and SUP-B15 were purchased from the American Type Culture Collection. All these cell lines were cultured in RPMI1640 (Gibco, Thermo Fisher Scientific, Waltham, MA, USA) containing 10\% FBS (Gibco), $100 \mathrm{U} / \mathrm{mL}$ penicillin, and $100 \mu \mathrm{g} / \mathrm{mL}$ streptomycin (HyClone, Logan, UT, USA). The human fibroblast cell line, BJ, and human hepatic cell line, L-02, were purchased from the American Type Culture Collection and cultured in DMEM (Gibco) containing 10\% FBS and penicillin/streptomycin. All the cell lines were maintained in a $5 \% \mathrm{CO}_{2}$ humidified incubator at $37^{\circ} \mathrm{C}$ according to the instructions from the sources.

\section{Virus construction, replication, purification, and titer determination}

The plasmid pDC316-PDCD5 was kindly provided by Professor Da-Long Ma (Peking University, Beijing). The plasmids pClon15, PENTR11-Linker, and pPE3-F11B were purchased from Microbix Biosystems Inc. (Toronto, ON, Canada). The pSG600 vector was kindly provided by the Laboratory of Viral and Gene Therapy, Eastern Hepatobiliary Surgical Hospital (Shanghai, China).

The PDCD5 gene was released from pDC316-PDCD5 using EcoRI digestion and then sequenced for confirmation. Then, PDCD5 cDNA was cloned into plasmid pClon 15 in the forward orientation, and the PDCD5 fragment was released from pClon 15 and ligated with PENTR11-Linker. The obtained novel adenoviral vector, named PENTR11PDCD5, was then ligated with pPE3-F11B to yield a novel plasmid, pPE3-F11B-PDCD5. This plasmid was cotransfected with pSG600 into HEK293 cells using Lipofectamine 2000 (Invitrogen ${ }^{\mathrm{TM}}$, Waltham, MA, USA). By homologous recombination in HEK293 cells, the novel CRAd SG611PDCD5 was generated. Further, the CRAd vector SG611-null and SG611-EGFP containing the enhanced green fluorescent protein were similarly constructed as a null control and marker control, respectively. 
For viral replication, HEK293 cells were infected with SG611-PDCD5, incubated at $37^{\circ} \mathrm{C}$ in $5 \% \mathrm{CO}_{2}$, collected, and routinely purified when replicating to the adequate volume. Finally, the viral titer was determined and was found to be $1.44 \times 10^{10} \mathrm{TCID} 50 / \mathrm{mL}$ for the stock, as required. ${ }^{14}$

\section{Cell-counting kit-8 (CCK-8) proliferation assay}

Cell viability was measured using CCK-8 (Dojindo, Japan). Leukemic cells were plated at a density of $1 \times 10^{4}$ per well in 96-well plates. The cells were infected with SG611-PDCD5 at a multiplicity of infection (MOI) of 48 hours and then treated with daunorubicin for 24 hours (Cayman Chemical, Shanghai Local Agent, China). The concentration gradients of daunorubicin and SG611-PDCD5, as well the ratios of the two agents, were designed in accordance with the Chou-Talalay method..$^{15}$ Next, $10 \mu \mathrm{L}$ of CCK-8 reagent was added to each well. After $1.5-3$ hours of incubation at $37^{\circ} \mathrm{C}$, the absorbance was measured at $450 \mathrm{~nm}$ on a scanning multi-well spectrophotometer (Bio-Rad, Hercules, CA, USA). The relative levels of cell proliferation in each group of cells were calculated according to the formula, cell survival rate $(\%)=($ mean OD value of treated cells - mean OD value of untreated cells)/(mean OD value of untreated cells-mean OD value of culture medium) $\times 100$. The mean OD value of each group was measured from three wells.

\section{Annexin- $\mathrm{V}$ apoptosis assays}

K562 and BV-173 cells were harvested in the exponential growth phase and plated at a density of $5 \times 10^{5}$ per well in 24-well plates. Cells were transfected with SG611-PDCD5 at different MOIs (40, 80, and $120 \mathrm{pfu} / \mathrm{cell})$ for 24 hours and then with $0.01,0.03$, and $0.1 \mu \mathrm{g} / \mathrm{mL}$ daunorubicin for an additional 24 hours. Next, the number of apoptotic cells was measured using an FACSCanto II flow cytometer (BD Bioscience, Franklin Lakes, NJ, USA) and annexin-V-PE and 7-amino-actinomycin D (7-AAD) staining (Annexin V-PE Apoptosis Detection Kit; BD Pharmingen, Franklin Lakes, NJ, USA) as recommended by the manufacturer. Briefly, cells were washed twice with ice-cold PBS and incubated with $100 \mu \mathrm{L}$ binding buffer, $5 \mu \mathrm{L}$ annexin-V-PE, and $5 \mu \mathrm{L}$ 7-AAD for 15 minutes at room temperature in the dark. Next, $400 \mu \mathrm{L}$ of binding buffer was added and early (annexin-V-PE positive) and late (annexin-V-PE and 7-AAD positive) apoptotic cells were identified by flow cytometry.

\section{Animal experiments}

Female BALB/c nu/nu mice (4-5 weeks of age; Experimental Animal Center, Peking University Health Science Center,
Beijing, China) were used in this study. The experimental animals were maintained and handled with the approval of the Institutional Authority for Laboratory Animal Care of Peking University. All animal experiments also strictly abided by the Beijing Ethical Examination Guidelines for Experimental Animal Welfare and Beijing Experimental Animal Management Regulations for the welfare of the experimental animals. Mice were pretreated with intraperitoneal (i.p.) injections of cyclophosphamide once daily at a dose of $100 \mathrm{mg} / \mathrm{kg}$ for two consecutive days. Two days later, $1.0 \times 10^{7} \mathrm{~K} 562$ cells in a total volume of $80 \mu \mathrm{L}$ were injected subcutaneously (s.c.) into the right flank of the mice. When the diameter of the tumor exceeded 5-7 mm, the mice were randomly divided into four groups as follows: seven mice in the control group and nine mice each in the daunorubicin group, SG611-PDCD5 group, and the daunorubicin plus SG611-PDCD5. The latter two groups were administered a total of $1 \times 10^{9} \mathrm{pfu}$ of the virus five times via intratumoral injections, once every other day. Then, 24 hours later, the mice were administered $400 \mu \mathrm{g} / \mathrm{kg}$ daunorubicin i.p. every 2 days a total of four times for the daunorubicin group and daunorubicin plus SG611-PDCD5 group, with $0.9 \%$ saline as a control. Tumor sizes were monitored every other day. Tumor volume was calculated using the following formula:

Maximal diameter $\times$ Perpendicular diameter ${ }^{2} \times 0.52$.

\section{Terminal deoxynucleotidyl transferase dUTP nick end labeling (TUNEL) assay}

Apoptosis in tumor tissues was detected using the TUNEL assay (TUNEL kit; Intergen Co. Ltd., Burlington, MA, USA) according to the manufacturer's instructions. Briefly, samples were digested with $200 \mathrm{mg} / \mathrm{L}$ proteinase $\mathrm{K}$ at $37^{\circ} \mathrm{C}$ for 20 minutes. Then, $20 \mu \mathrm{L}$ of the kit reaction agent was added to each sample according to the kit manual, and paraffin-covered slides were incubated at $37^{\circ} \mathrm{C}$ for 60 minutes in a humidified chamber. Next, $2 \mu \mathrm{L}$ terminal deoxynucleotidyl transferase and $18 \mu \mathrm{L}$ nucleotide mixture containing biotinylated UTP were added to the reactions. The slides were washed twice with phosphate buffer, and each slide was incubated with $50 \mu \mathrm{L}$ SP-AP (Promega, Madison, WI, USA) at $37^{\circ} \mathrm{C}$ for 1 hour. Sections were visualized with BCIP/NBT (5-bromo-4-chloro-3-indolyl phosphate/nitro blue tetrazolium) staining using a light microscope, and nuclei of positive cells appeared blue-black. Images were acquired at 400× magnification, and three different fields were examined per slide. 


\section{Statistical analysis}

SPSS 19.0 software (Chicago, IL, USA) was used for statistical analysis. Data from in vitro experiments were assessed using the Student's $t$-test, whereas those from in vivo experiments were examined using independent-samples $t$-test for two groups and one-way ANOVA for more than two groups. Results were given as the mean $\pm \mathrm{SD}$, and differences with $P<0.05$ were considered statistically significant. The half-maximal inhibitory concentrations (IC50) were calculated using GraphPad Prism Inc., (San Diego, CA, USA). Calcusyn 2.1 (Biosoft, San Diego, CA, USA) software was used to investigate the combined effects of SG611PDCD5 and daunorubicin. The combination index (CI) and dose reduction index (DRI) were used to demonstrate the combined effects and dose reduction when the two agents were combined, respectively. $\mathrm{CI}<1$ suggested a synergistic effect, $\mathrm{CI}=1$ suggested an additive effect, $\mathrm{CI}>1$ suggested an antagonistic effect, and DRI $>1$ suggested that the dose could be reduced when the two agents were combined. ${ }^{15}$
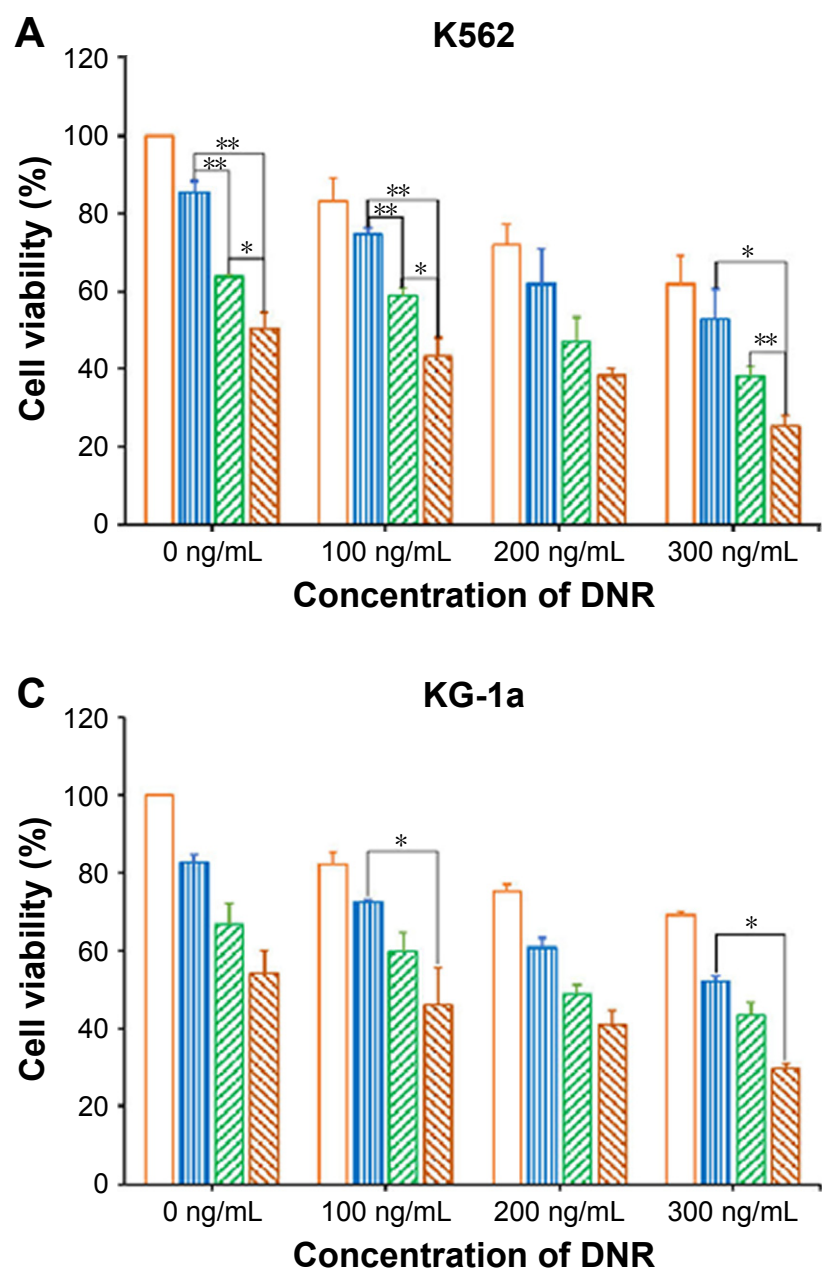

\section{Results}

\section{SG6II-PDCD5 and daunorubicin}

\section{synergistically inhibited proliferation}

The K562, MEG-01, KG-1a, HL-60, SUP-B15, and BV-173 cell lines were infected with SG611-PDCD5 at an MOI of $0,40,80$, and $120 \mathrm{pfu} / \mathrm{cell}$ for 24 hours, followed by daunorubicin at different doses for another 24 hours. The CCK- 8 assay was performed 72 hours later. As shown in Figure 1, the cell viabilities decreased as the dose of SG611-PDCD5 and daunorubicin increased, showing the synergistic effect of these agents in inhibiting the proliferation of the cell lines in a dose-dependent manner. The viability of K562 cells treated with SG611-PDCD5 at an MOI of $120 \mathrm{pfu} / \mathrm{cell}$ in combination with $0.30 \mu \mathrm{g} / \mathrm{mL}$ daunorubicin was $25.29 \% \pm 2.90 \%$, and this was significantly lower than that of cells treated with the same doses of SG611-PDCD5 (50.38\% $\pm 4.12 \%)$ or daunorubicin $(61.79 \% \pm 5.73 \%)$ used alone. However, as demonstrated in Figure S1, the viability of the healthy BJ
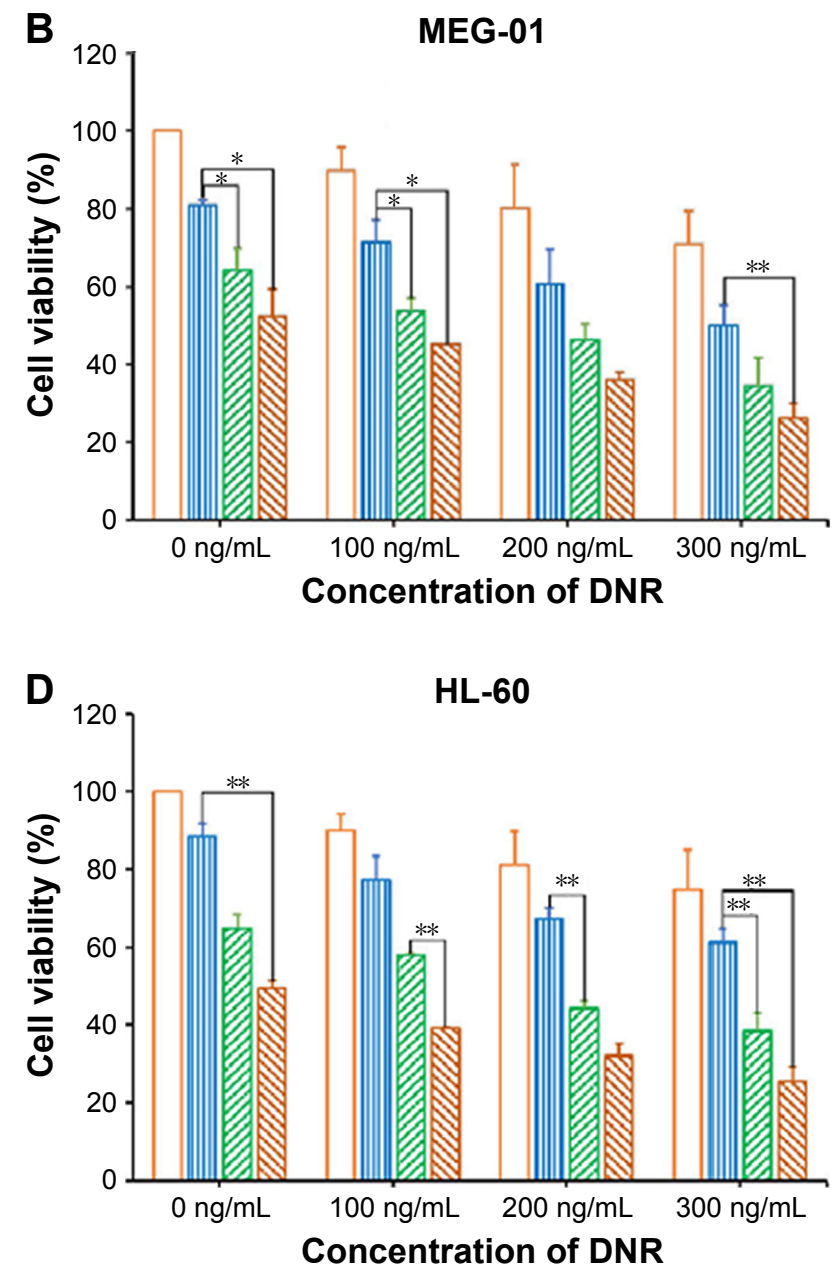

$\square 0$ 四 $40 \mathrm{pfu} / \mathrm{cell} \quad \square 80 \mathrm{pfu} / \mathrm{cell} \quad \mathbb{\$} 120 \mathrm{pfu} / \mathrm{cell}$

Figure I (Continued) 

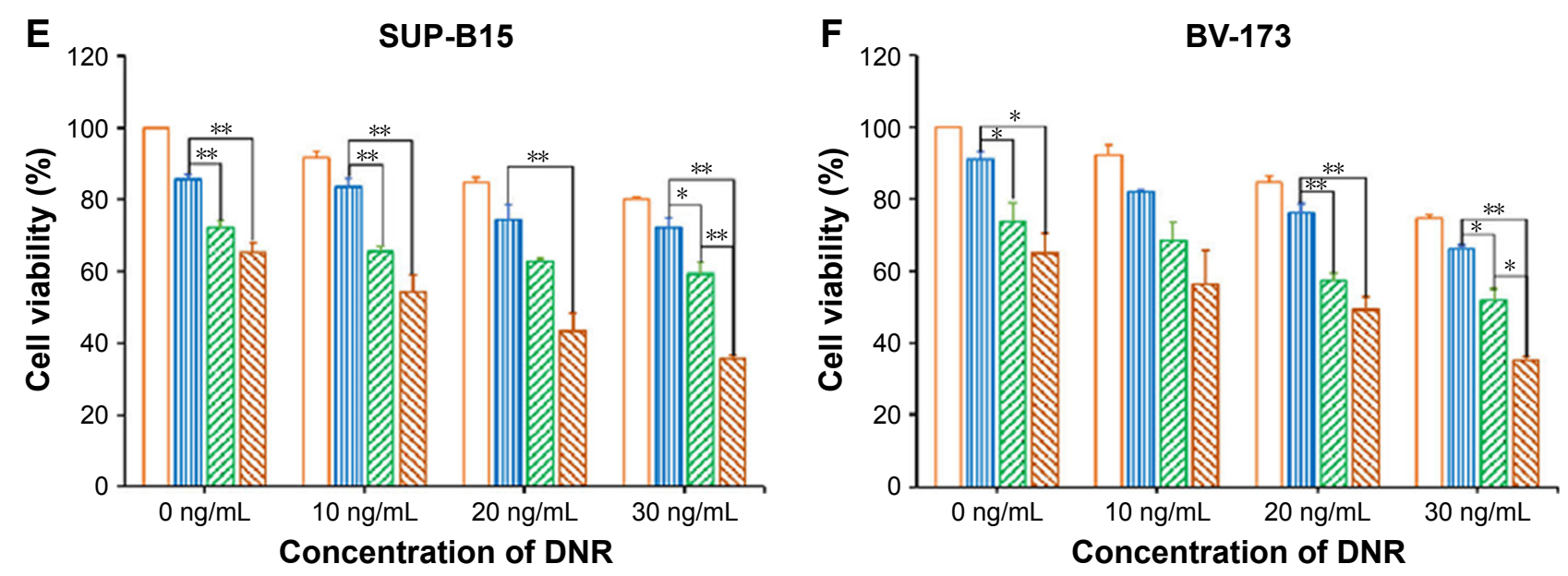

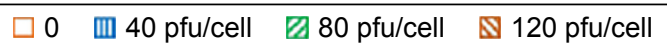

Figure I Cytotoxic effects as determined by a CCK-8 assay in a panel of leukemia cells after treatment with SG6 I I-PDCD5 plus daunorubicin compared to SG6 I I-PDCD5 or daunorubicin alone.

Notes: The results showed the effect on K562 (A), MEG-0I (B), KG-Ia (C), HL-60 (D), SUP-BI5 (E) and BV-I73 cells (F), respectively and that the cell viabilities decreased to a greater extent in the combined treatment group than in the SG6II-PDCD5 or daunorubicin alone groups. $* P<0.05, * * P<0.01$.

Abbreviations: CCK-8, Cell Counting Kit-8; DNR, daunorubicin.

cell line and human hepatic L-02 cells treated with even 1,000 pfu/cell SG611-PDCD5 for 72 hours remained relatively high $(\sim 68.17 \% \pm 2.69 \%$ and $74.93 \% \pm 4.78 \%$, respectively), suggesting that SG611-PDCD5 specifically targets tumors, and thereby implying its safety.
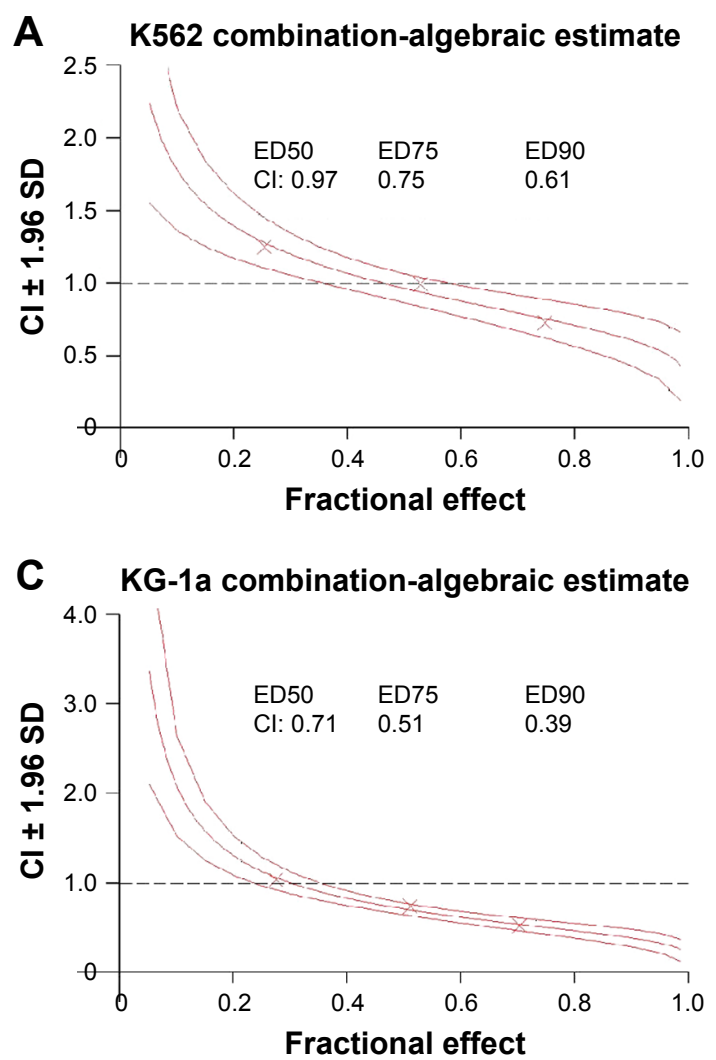

To investigate the combination effect of SG611-PDCD5 and daunorubicin, we used the Chou-Talalay method, as previously reported. ${ }^{15}$ As shown in Figure 2A, we found that the $\mathrm{CI}<1$ when the proliferative inhibition ratio was $>0.50$, suggesting that, when the MOI of SG611-PDCD5
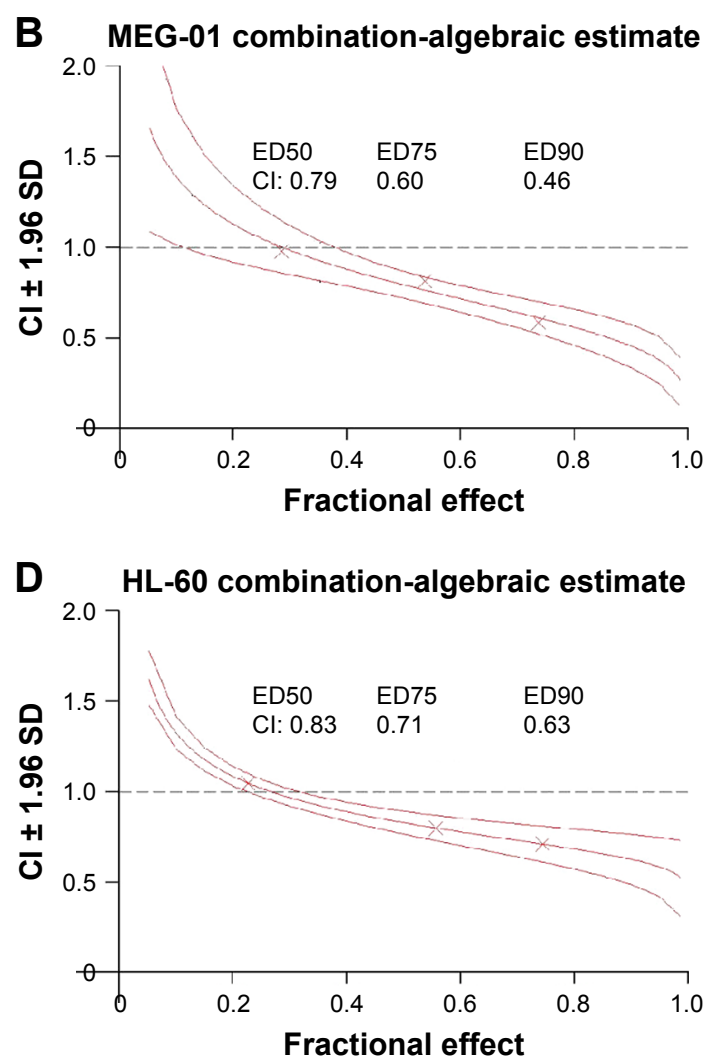

Figure 2 (Continued) 

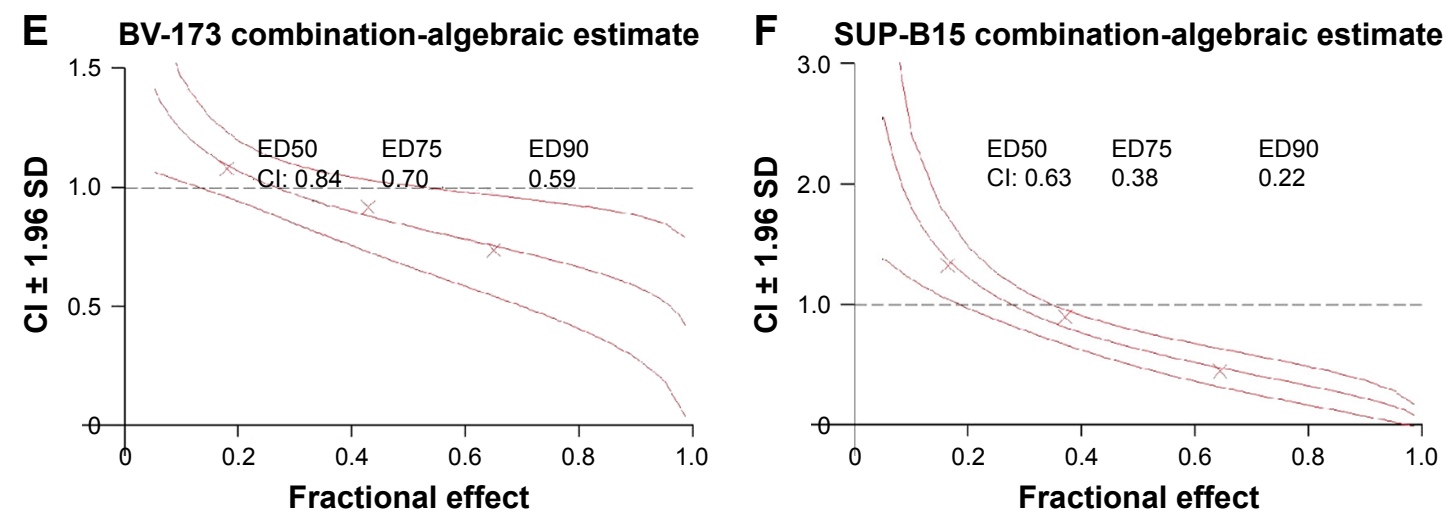

Figure 2 A Chou-Talalay analysis demonstrated that SG6I I-PDCD5 and low-dose daunorubicin have a synergistically anti-proliferative effect on various leukemic cell lines. Notes: The effects of the various treatments are demonstrated on (A) K562, (B) MEG-0I, (C) KG-Ia, (D) HL-60, (E) BV-I73, and (F) SUP-BI 5 cells, respectively. As shown in (A), the $\mathrm{Cl}<\mathrm{I}$ when the proliferative inhibition ratio was $>0.50$, suggesting a synergistic effect on $\mathrm{K} 562$ cells under these conditions. ED50, ED75, and ED90 referred to the dose when the fractional effect was $0.50,0.75$, and 0.90 , respectively.

Abbreviation: $\mathrm{Cl}$, combination index.

was $80 \mathrm{pfu} / \mathrm{cell}$ and the concentration of daunorubicin was $0.20 \mu \mathrm{g} / \mathrm{mL}$ or $0.30 \mu \mathrm{g} / \mathrm{mL}$, or when the MOI of SG611PDCD5 was $120 \mathrm{pfu} / \mathrm{cell}$ with the concentration daunorubicin at $0.10-0.30 \mu \mathrm{g} / \mathrm{mL}$, these two agents exhibited a synergistic effect on K562 cells. We further studied whether the combination of the two agents could reduce the dose of daunorubicin. As a result, we found that the DRI $>1$ when the proliferation inhibition ratio was $>0.10$, as shown in Figure 3. This finding suggests that, when the two agents were combined, the dose of daunorubicin could be reduced in most cases. Similar results were also found using other cell lines, as demonstrated in Figures 2B-F and 3 . We also provided the dose curves and IC-50 of daunorubicin in leukemic cell lines, including K562, MEG-01, KG-1a, HL-60, SUP-B15, and BV-173, as presented in Figure S2. These results provide a promising implication for reducing the dose-related toxicity of daunorubicin in a clinical setting.

\section{SG6 I I-PDCD5 and daunorubicin synergistically promote apoptosis}

To measure the apoptosis induced by SG611-PDCD5 and daunorubicin, in combination, we studied their effect on K562 and BV-173 cells using annexin-V and 7-AAD staining and flow cytometry. As shown in Figure 4, compared with the control group, $\mathrm{K} 562$ cells treated with $0.01 \mu \mathrm{g} / \mathrm{mL}$ daunorubicin did not show considerable apoptosis, but the extent of apoptosis was significantly higher in cells treated with this dose of daunorubicin combined with SG611-PDCD5 at an MOI of 40 or 80 pfu/cell. SG611-PDCD5 and daunorubicin promoted

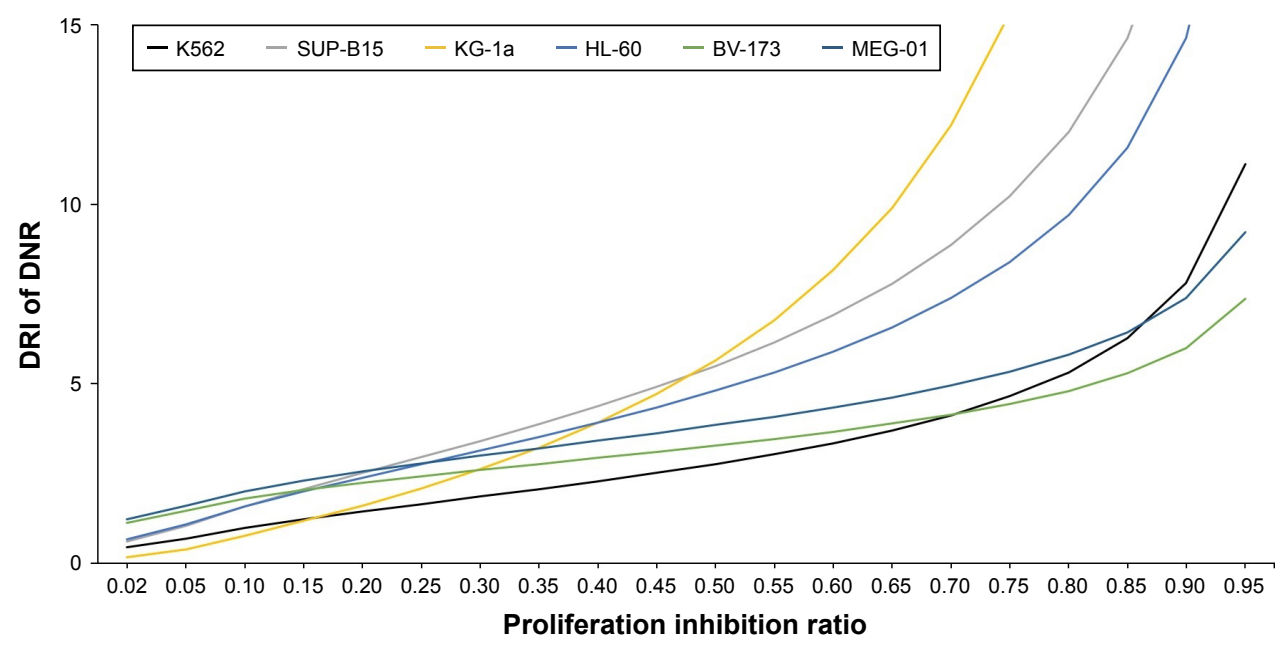

Figure 3 The DRI of daunorubicin when combined with SG6II-PDCD5 in the leukemic cell lines.

Notes: As demonstrated in the K562 cell line, the DRI of daunorubicin was $>$ I when the proliferation inhibition ratio was $>0$. I, suggesting that, when the two agents were combined, the dose of daunorubicin could be reduced in most cases. Similar results were observed in the other cell lines as demonstrated.

Abbreviations: DRI, dose reduction index; DNR, daunorubicin. 

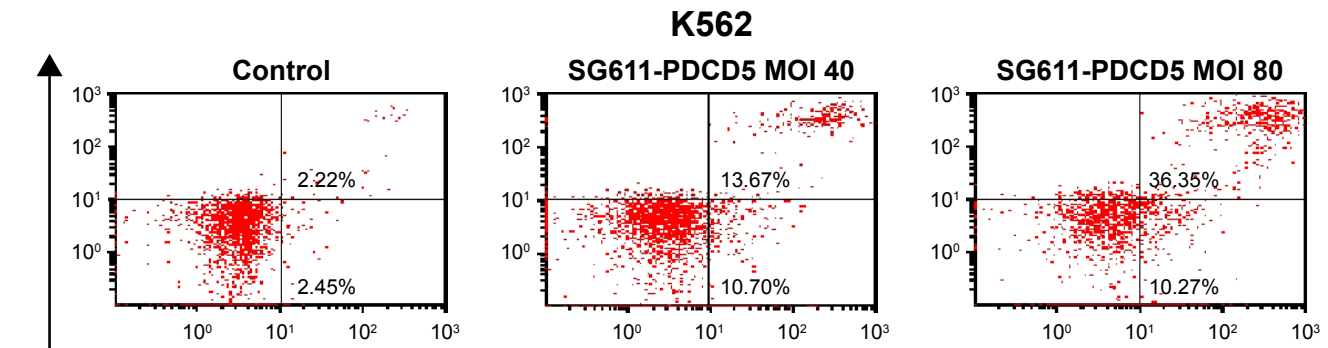

SG611-PDCD5 MOI 40+ DNR $0.01 \mu \mathrm{g} / \mathrm{mL}$
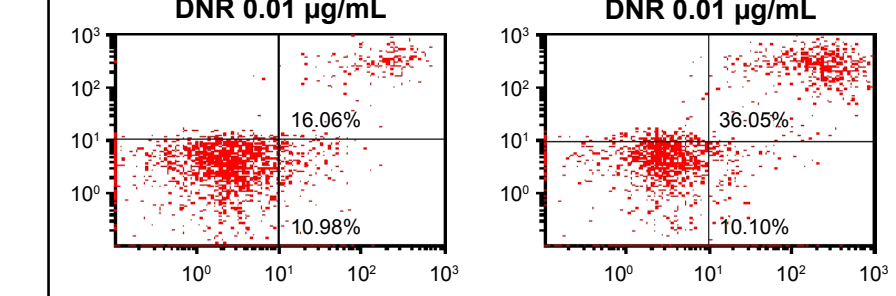

SG611-PDCD5 MOI 80+ DNR $0.01 \mu \mathrm{g} / \mathrm{mL}$

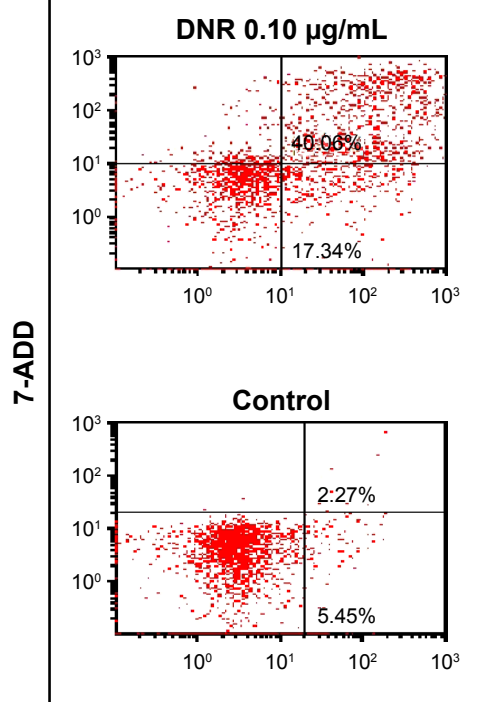

SG611-PDCD5 MOI 40+ DNR $0.10 \mu \mathrm{g} / \mathrm{mL}$

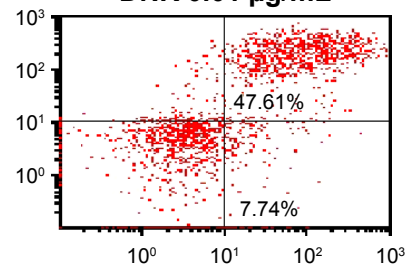

SG611-PDCD5 MOI 80+

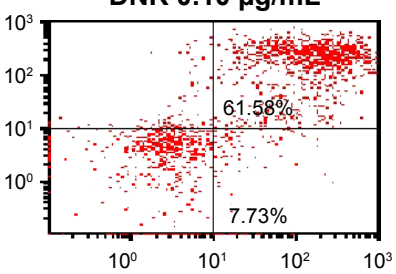
DNR $0.10 \mu \mathrm{g} / \mathrm{mL}$

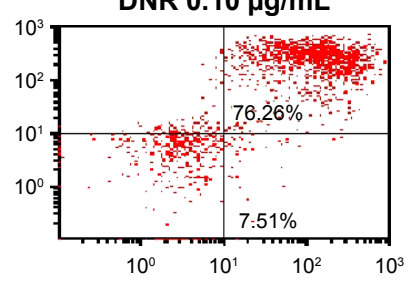

\section{BV-173}
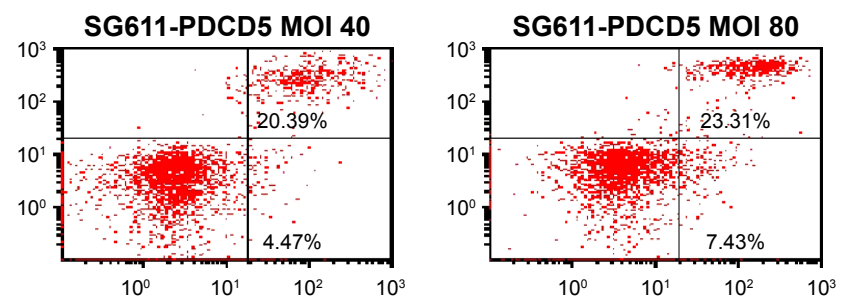

SG611-PDCD5 MOI 40+ DNR $0.01 \mu \mathrm{g} / \mathrm{mL}$
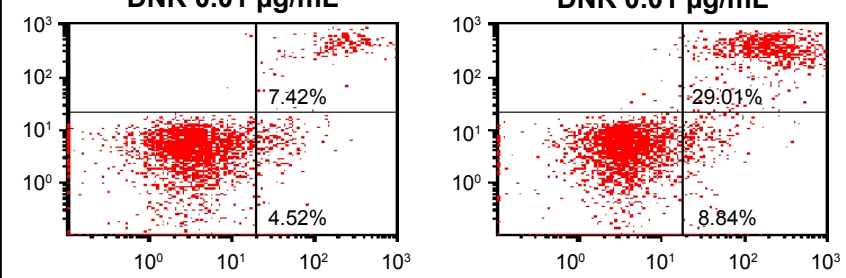

SG611-PDCD5 MOI 80+ DNR $0.01 \mu \mathrm{g} / \mathrm{mL}$

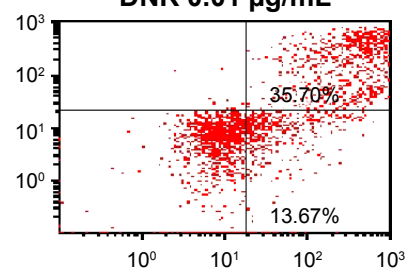

SG611-PDCD5 MOI 40+ DNR $0.10 \mu \mathrm{g} / \mathrm{mL}$
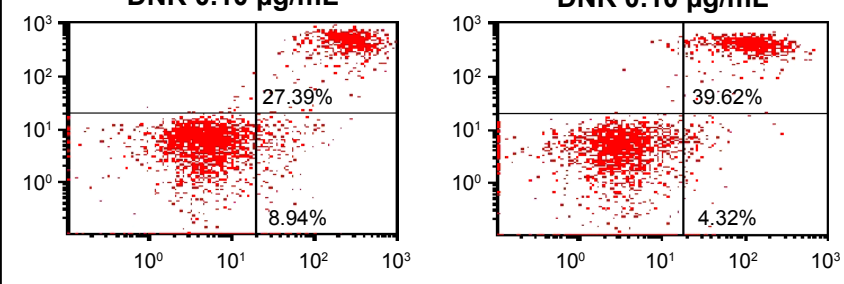

SG611-PDCD5 MOI 80+ DNR $0.10 \mu \mathrm{g} / \mathrm{mL}$

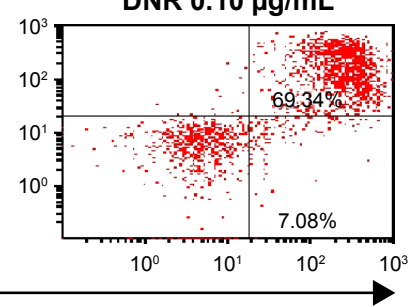

Annexin-V

Figure 4 Apoptosis of K562 and BV-173 cells treated with SG6II-PDCD5 followed by daunorubicin treatment.

Notes: The combination of SG6I I-PDCD5 and low-dose daunorubicin exhibited enhanced proapoptosis efficacy over than that of SG6| I-PDCD5 or daunorubicin alone. The percentage of apoptotic cells following treatment with SG6II-PDCD5 (MOI=80 pfu/cell), in combination with DNR $(0.10 \mu g / \mathrm{mL})(72.38 \% \pm 8.54 \%)$, was significantly higher than that of SG6II-PDCD5 $(43.08 \% \pm 3.57 \%)$ or daunorubicin $(44.01 \% \pm 12.56 \%)$ alone $(P=0.02627$ and $P=0.00652$, respectively).

Abbreviations: MOI, multiplicity of infection; DNR, daunorubicin. 
apoptosis in tumor cells synergistically in a dose-dependent manner, when the MOI of SG611-PDCD5 ranged from 0 to $80 \mathrm{pfu} / \mathrm{cell}$ and the dose of daunorubicin ranged from 0.00 to $0.10 \mu \mathrm{g} / \mathrm{mL}$. Similar results were observed in BV-173 cells and it seemed that these lymphoblastic leukemic cells were more sensitive to the proapoptotic effects of SG611-PDCD5 than the myelogenous leukemic K562 cells.

\section{In vivo antitumor efficacy of SG6 I I- PDCD5 and low-dose daunorubicin}

To compare the antitumor efficacy of SG611-PDCD5 in combination with low-dose daunorubicin, a K562 tumor xenograft model was successfully established in nude mice, as discussed above and as reported previously. ${ }^{13,16}$ As shown in Figure 5, compared with the control group, the group treated with daunorubicin showed suppressed tumor growth on days 10 $(P=0.013)$ and $12(P=0.040)$ after treatment. Although SG611PDCD5 treatment alone and in combination resulted in significant tumor suppression on days $6,8,10$, and $12(P<0.01$ for all), the combined treatment showed better antitumor efficacy than either agent alone on day 10 ( $P<0.05$ for all). These results suggest that local systemic SG611-PDCD5 injection plus daunorubicin treatment could effectively suppress tumor growth in vivo, and tumor inhibition with the combined treatment was superior to that with local SG611-PDCD5 injection or i.p. daunorubicin alone.

\section{Combined treatment with SG6 I I- PDCD5 and daunorubicin demonstrates proapoptotic effects in vivo}

Tumors were excised on day 12 after treatment, and we examined the percentage of apoptotic cells in the

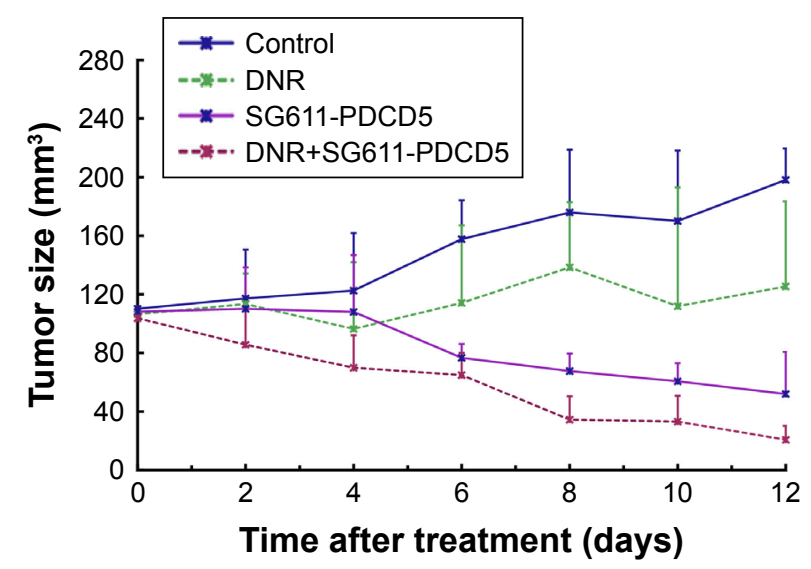

Figure 5 In vivo anti-proliferative effects of systemic treatment with SG6 I I-PDCD5 combined with low-dose daunorubicin.

Notes: Tumors were measured on days 2-12 after treatment. The results were expressed as mean $\pm S D$. Compared with the control group, the combined treatment group showed a significantly higher anti-proliferative effect on days 10 and 12 . Abbreviation: DNR, daunorubicin. treated tumors by using the TUNEL assay. As shown in Figure 6, significantly more apoptotic cells were observed in the SG611-PDCD5 plus daunorubicin group than in the SG611-PDCD5 or daunorubicin groups $(25.00 \pm 0.82$, $12.67 \pm 2.05$, and $7.67 \pm 2.49$ apoptotic cells/field, respectively; $P<0.001)$. These results suggested that SG611-PDCD5 and daunorubicin synergistically promote apoptosis in tumor cells in vivo.

\section{Discussion}

PDCD5 is an apoptosis-regulating gene identified in TF-1 cells undergoing apoptosis, and its expression has been found to be decreased in certain malignancies such as hepatocellular carcinoma, ${ }^{17}$ breast cancer, ${ }^{18,19}$ gastric cancer, ${ }^{20}$ astrocytic gliomas, ${ }^{21}$ suggesting that this gene plays an important role in cancer pathogenesis and/or disease progression. Zhang et $\mathrm{al}^{10}$ found that human recombinant PDCD5 protein had pro-apoptotic effects on the HL-60 leukemic cell line and enhanced the apoptosis in hepatocarcinomatous cells induced by hydroxycamptothecin. We previously found lower expression levels of PDCD5 in both AML and CML, respectively, marrow cells than those in normal donor marrow cells, and we also identified a negative correlation between the relative levels of PDCD5 and BCR/ABL expression in all CML patients. ${ }^{8}$ Additionally, we demonstrated that adenovirusmediated PDCD5 gene transfer could enhance the apoptosis in $\mathrm{K} 562$ cells induced by etoposide and idarubicin, both in vitro and in an in vivo nude mouse model..$^{22,23}$

The adenovirus vector system is currently one of the most widely used viral vectors in gene function studies, gene-transfer therapies, vaccine development, and many other fields. The first adenovirus system, a replication defective vector, was established in 1999 by the father of the adenovirus vector, Frank Grahamset. CRAds are oncolytic adenoviruses that have selective replicative ability in tumor cells, and they have been proved to be more effective for gene therapy for some malignancies with potent antitumor ability. $9,10,24$

Benefiting from all these efforts in the field of oncolytic adenoviruses and our understanding of the function of $P D C D 5$, we constructed a novel triple-regulated CRAd carrying the PDCD5 gene expression cassette SG611-PDCD5, in which the E1 a gene with 24 nucleotides deleted from the CR2 region is controlled by the hTERT promoter, the $E 1 b$ gene is controlled by the HRE promoter, and PDCD5 is controlled by the cytomegalovirus promoter. The innovative design of SG611-PDCD5 allows it to selectively target tumors. We previously showed that SG611-PDCD5 treatment alone can effectively suppress the growth of the human leukemic 
A

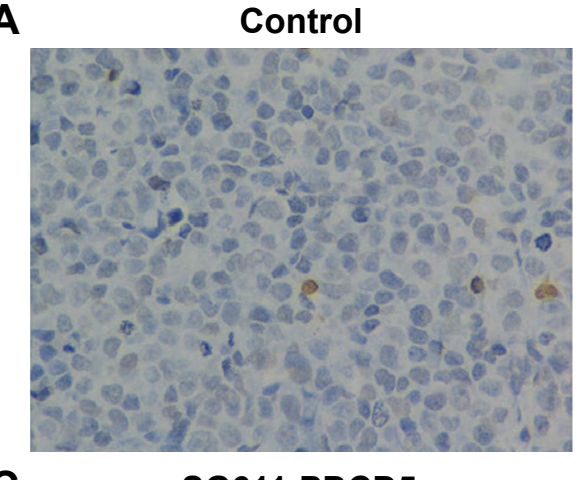

C

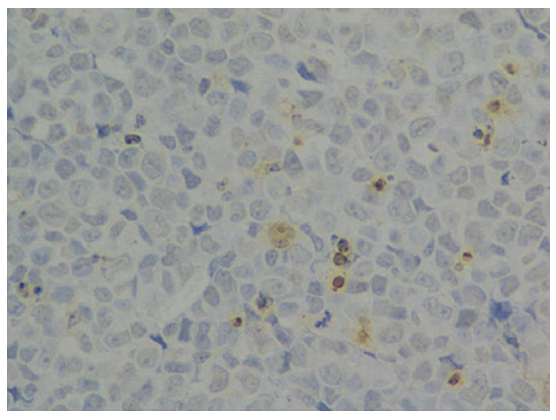

B

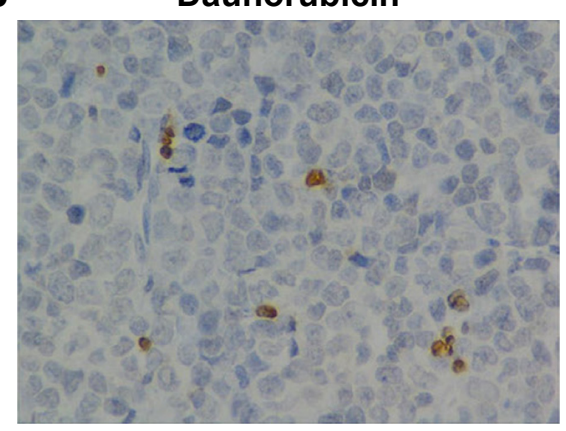

D SG611-PDCD5+Daunorubicin

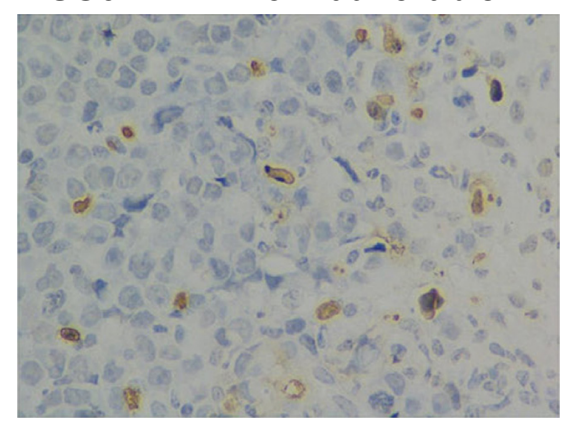

Figure 6 In vivo proapoptotic effect of SG6I I-PDCD5 combined with low-dose daunorubicin.

Notes: Apoptotic cells in excised tumors after different treatments were detected by TUNEL staining. Cells stained blue-black were apoptotic cells. The number of apoptotic cells per field in the control (A), DNR (B), SG6II-PDCD5 (C), and SG6II-PDCD5 plus DNR (D) treated groups were 4.67 \pm I.25, 7.8 $\pm 2.67,12.50 \pm 2.27$, and $25.00 \pm 0.82$, respectively. A greater number of apoptotic cells per field were observed in the SG6II-PDCD5 plus DNR group compared with the SG6II-PDCD5 or DNR group $(P=0.00682$ and $P=0.00588$, respectively). Magnification $\times 400$.

Abbreviation: DNR, daunorubicin.

cell lines K562, MEG-01, and NB4, and the K562 xenograft tumor model in nude mice. More importantly, its safety and tumor inhibition ability were superior to those of SG611 or the replication-defective Ad-PDCD5. 13,22,23

Daunorubicin is a classical anthracycline that plays a significant role in the treatment of patients with hematologic malignancies and is widely recommended for both children and adults with leukemia. Unfortunately, the use of daunorubicin is limited by its dose-dependent and cumulative toxicity, especially cardiotoxicity, manifested as dilated cardiomyopathy with or without symptoms of heart failure. This limitation brings a considerable challenge for clinicians, especially those treating the elderly, pediatric leukemic patients, or patients with recalcitrant disease. . $^{3,25}$

In the present study, we evaluated the synergetic effect of SG611-PDCD5 and daunorubicin via in vitro and in vivo experiments. In a panel of leukemia cells, combined treatment with SG611-PDCD5 and daunorubicin inhibited cell proliferation to a greater extent than treatment with SG611-PDCD5 or daunorubicin alone did. SG611-PDCD5 treatment enhanced the apoptosis induced by low-dose daunorubicin to a higher extent than the extent in cells treated with SG611-PDCD5 or daunorubicin alone. In a nude mouse model with K562 tumor xenografts, combined treatment with SG611-PDCD5 and daunorubicin showed significantly better antitumor efficacy than daunorubicin or SG611-PDCD5 alone on days 8 and 10 after treatment. Pathologic analysis showed a higher percentage of apoptotic cells in the group that received the combined treatment than in those that received either treatment alone. Collectively, the findings suggested that SG611-PDCD5 enhanced the antitumor effects of the traditional chemotherapeutic daunorubicin. Combined treatment with SG611-PDCD5 and daunorubicin could be adapted to clinical leukemia therapy, ensuring more potent antitumor effects and lower associated systemic toxicity.

In summary, we showed that SG611-PDCD5 and daunorubicin have synergistic anti-proliferative and proapoptotic effects, not only in vitro but also in vivo. Our findings may lead to the development of gene viral therapy with potent antitumor effects and limited adverse effects compared to traditional chemotherapy, thereby providing new therapeutic strategies for leukemia. Further investigations are required to further clarify the specific molecular mechanisms responsible for the observed synergy between these two agents, and increase the efficacy, safety, and clinical feasibility of this combined treatment. 


\section{Acknowledgment}

This work was supported by the National Natural Science Foundation of China [Grant numbers 81570182, 81770156], the Foundation for Innovative Research Groups of the National Natural Science Foundation of China [grant number 81621001], and the Key Program of National Natural Science Foundation of China [Grant number 81530046].

\section{Disclosure}

The authors report no conflicts of interest in this work

\section{References}

1. Hanahan D, Weinberg RA. The hallmarks of cancer. Cell. 2000;100(1): 57-70.

2. Lacasse EC, Baird S, Korneluk RG, Mackenzie AE. The inhibitors of apoptosis (IAPs) and their emerging role in cancer. Oncogene. 1998; 17(25):3247-3259.

3. Valcovici M, Andrica F, Serban C, Dragan S. Cardiotoxicity of anthracycline therapy: current perspectives. Arch Med Sci. 2016;12(2):428-435.

4. Miyake H, Hara I, Hara S, Arakawa S, Kamidono S. Synergistic chemosensitization and inhibition of tumor growth and metastasis by adenovirus-mediated P53 gene transfer in human bladder cancer model. Urology. 2000;56(2):332-336.

5. Tuve S, Racek T, Niemetz A, et al. Adenovirus-mediated TA-p73beta gene transfer increases chemosensitivity of human malignant melanomas. Apoptosis. 2006;11(2):235-243.

6. Okumura K, Nakase M, Nakamura S, et al. Bax gene therapy for human osteosarcoma using cationic liposomes in vivo. Oncol Rep. 2007;17(4): 769-773.

7. Wang X, Su C, Cao H, et al. A novel triple-regulated oncolytic adenovirus carrying $\mathrm{p} 53$ gene exerts potent antitumor efficacy on common human solid cancers. Mol Cancer Ther. 2008;7(6):1598-1603.

8. Ruan GR, Qin YZ, Chen SS, et al. Abnormal expression of the programmed cell death 5 gene in acute and chronic myeloid leukemia. Leuk Res. 2006;30(9):1159-1165.

9. Su CQ, Sham J, Xue HB, et al. Potent antitumoral efficacy of a novel replicative adenovirus $\mathrm{CNHK} 300$ targeting telomerase-positive cancer cells. J Cancer Res Clin Oncol. 2004;130(10):591-603.

10. Zhang Q, Chen G, Peng L, et al. Increased safety with preserved antitumoral efficacy on hepatocellular carcinoma with dual-regulated oncolytic adenovirus. Clin Cancer Res. 2006;12(21):6523-6531.
11. Shay JW, Wright WE. Telomeres and telomerase: implications for cancer and aging. Radiat Res. 2001;155(1 Pt 2):188-193.

12. Hewitson KS, Schofield CJ. The HIF pathway as a therapeutic target. Drug Discov Today. 2004;9(16):704-711.

13. Xie M, Niu JH, Chang Y, et al. A novel triple-regulated oncolytic adenovirus carrying PDCD5 gene exerts potent antitumor efficacy on common human leukemic cell lines. Apoptosis. 2009;14(9):1086-1094.

14. Labarre DD, Lowy RJ. Improvements in methods for calculating virus titer estimates from TCID50 and plaque assays. J Virol Methods. 2001;96(2):107-126.

15. Chou TC. Drug combination studies and their synergy quantification using the Chou-Talalay method. Cancer Res. 2010;70(2):440-446.

16. Zhou J, Yao QM, Li JL, et al. Synergistic antitumor activity of tripleregulated oncolytic adenovirus with VSTM1 and daunorubicin in leukemic cells. Apoptosis. 2016;21(10):1179-1190.

17. Xu XR, Huang J, Xu ZG, et al. Insight into hepatocellular carcinogenesis at transcriptome level by comparing gene expression profiles of hepatocellular carcinoma with those of corresponding noncancerous liver. Proc Natl Acad Sci U S A. 2001;98(26):15089-15094.

18. Sack H. Gene expression profiles in hereditary breast cancer. Strahlenther Onkol. 2001;177(10):559-560.

19. Zucchi I, Mento E, Kuznetsov VA, et al. Gene expression profiles of epithelial cells microscopically isolated from a breast-invasive ductal carcinoma and a nodal metastasis. Proc Natl Acad Sci U S A. 2004; 101(52):18147-18152.

20. Yang YH, Zhao M, Li WM, et al. Expression of programmed cell death 5 gene involves in regulation of apoptosis in gastric tumor cells. Apoptosis. 2006;11(6):993-1001.

21. Li H, Wang Q, Gao F, et al. Reduced expression of PDCD5 is associated with high-grade astrocytic gliomas. Oncol Rep. 2008;20(3):573-579.

22. Ruan GR, Chen SS, Chang Y, et al. Adenovirus-mediated PDCD5 gene transfer sensitizes apoptosis of K562 cells induced by etoposide. Zhongguo Shi Yan Xue Ye Xue Za Zhi. 2007;15(5):936-940.

23. Ruan GR, Zhao HS, Chang Y, et al. Adenovirus-mediated PDCD5 gene transfer sensitizes K562 cells to apoptosis induced by idarubicin in vitro and in vivo. Apoptosis. 2008;13(5):641-648.

24. Garber K. China approves world's first oncolytic virus therapy for cancer treatment. J Natl Cancer Inst. 2006;98(5):298-300.

25. Lipshultz SE, Alvarez JA, Scully RE. Anthracycline associated cardiotoxicity in survivors of childhood cancer. Heart. 2008;94(4): 525-533. 


\section{Supplementary materials}

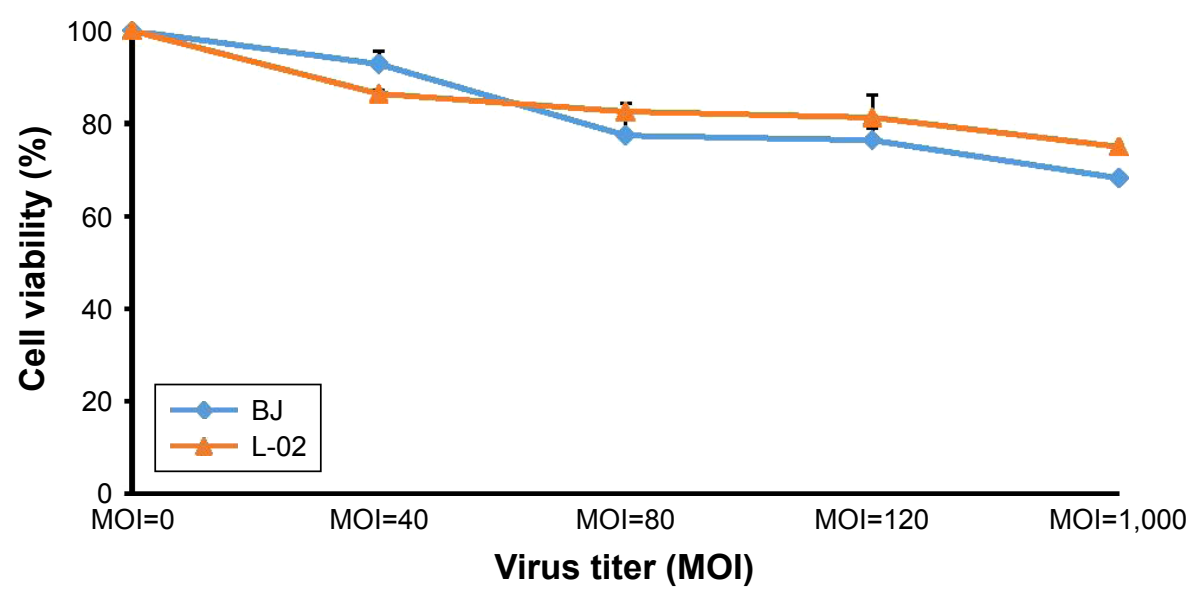

Figure SI Cytotoxic effects in the human fibroblast cell line, BJ, and human hepatic cell line, L-02, after infection with SG6 I I-PDCD5.

Notes: The viability of the healthy BJ and L-02 cells treated with as low as I,000 pfu/cell SG6II-PDCD5 for 72 hours remained relatively high ( $68.17 \%$ and $74.93 \%$, respectively), suggesting the relatively selective ability of SG6II-PDCD5. Abbreviation: MOI, multiplicity of infection.
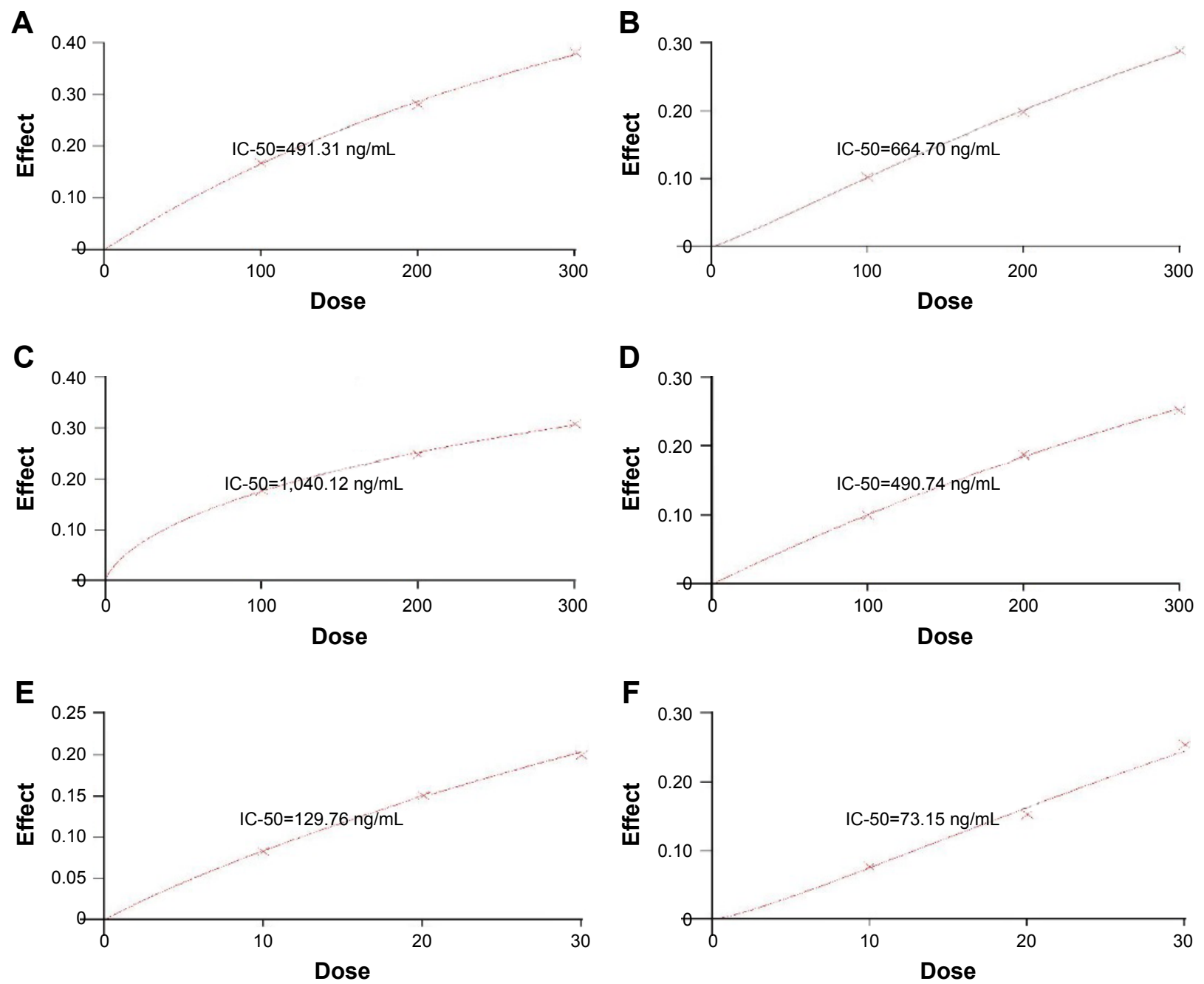

Figure S2 The dose curves and IC-50 of DNR in leukemic cell lines, including (A) K562, (B) MEG-0I, (C) KG-Ia, (D) HL-60, (E) SUP-BI5, and (F) BV-I73 cells. Abbreviation: DNR, daunorubicin. 


\section{Publish your work in this journal}

OncoTargets and Therapy is an international, peer-reviewed, open access journal focusing on the pathological basis of all cancers, potential targets for therapy and treatment protocols employed to improve the management of cancer patients. The journal also focuses on the impact of management programs and new therapeutic agents and protocols on

patient perspectives such as quality of life, adherence and satisfaction. The manuscript management system is completely online and includes a very quick and fair peer-review system, which is all easy to use. Visit http://www.dovepress.com/testimonials.php to read real quotes from published authors.

Submit your manuscript here: http://www.dovepress.com/oncotargets-and-therapy-journal 